\title{
Ectopic respiratory epithelial cell differentiation in bronchiolised distal airspaces in idiopathic pulmonary fibrosis
}

\author{
Laurent Plantier, ${ }^{1}$ Bruno Crestani, ${ }^{2,3}$ Susan E Wert, ${ }^{1}$ Monique Dehoux, ${ }^{3,4}$ \\ Barbara Zweytick, ${ }^{5}$ Andreas Guenther, ${ }^{6,7}$ Jeffrey A Whitsett ${ }^{1}$
}

\section{See Editorial, p 647}

- Additional figures and tables are published online only. To view these files please visit the journal online (http://thorax.bmj. com)

'Section of Neonatology, Perinatal and Pulmonary Biology, Perinatal Institute, Cincinnati Children's Hospital Medical Center, Cincinnati, Ohio, USA ${ }^{2}$ Assistance Publique-Hôpitaux de Paris, Hôpital Bichat, Service de Pneumologie A, Paris, France ${ }^{3}$ Inserm U 700, Université Paris Diderot-Paris 7, UFR de

Médecine-site Bichat, Paris, France

${ }^{4}$ Assistance Publique-Hôpitaux de Paris, Hôpital Bichat, Service de Biochimie A, Paris, France

${ }^{5}$ Department of Thoracic

Surgery, Vienna General

Hospital, Vienna, Austria

${ }^{6}$ University of Giessen Lung

Centre, Department of Internal

Medicine, Giessen, Germany

${ }^{7}$ Lung Clinic

Waldhof-Elgershausen,

Greifenstein, Germany

\section{Correspondence to}

Jeffrey A Whitsett, Division of Pulmonary Biology, Cincinnati Children's Hospital Medical Center, Perinatal Institute

MLC7029, 3333 Burnet Avenue, Cincinnati, OH 45229-3039, USA; jeff.whitsett@cchmc.org

Received 21 September 2010 Accepted 10 February 2011 Published Online First 21 March 2011

\section{ABSTRACT}

Background Bronchiolisation of distal airspaces is an unexplained feature of idiopathic pulmonary fibrosis (IPF). The authors sought to identify mechanisms driving the differentiation of mucus cells during the bronchiolisation process.

Methods Pathways governing airway mucus cell differentiation include SRY (sex determining region Y)box 2 (SOX2), Notch, forkhead box A3(FOXA3)/SAM pointed domain containing ETS transcription factor (SPDEF), epidermal growth factor (EGF) and the EGFrelated neuregulins NRG1 $\alpha$ and NRG1 $\beta$. Immunostaining for components of those pathways and mucins were performed on lung tissue obtained from patients with IPF $(n=20)$, chronic obstructive pulmonary disease $(n=13)$, idiopathic pulmonary artery hypertension $(n=5)$ and from organ donors $(n=6)$. NRG $1 \alpha$ and NRG1 $\beta$ were quantified in bronchoalveolar lavage fluid (BALF) of patients with early IPF $(n=20)$, controls $(n=9)$, and patients with other interstitial pneumonias $(n=13)$.

Results In IPF, the bronchiolised and enlarged distal airspaces stained for SOX2 are consistent with epithelial differentiation characteristic of conducting airway epithelium. IPF mucus cells expressed MUC5B but low levels of MUC5AC and MUC2, a profile typical of submucosal glands. Singularly, SPDEF, a transcription factor associated with mucus metaplasia, was rarely detected in mucus cells in IPF. The Notch target, HES1, was present in mucus cells from all groups. NRG1 $\alpha$ was detected in serous cells within normal submucosal glands and in epithelial cells lining honeycombing areas in IPF, and was not detected in other patients. NRG1 $\alpha$ concentrations were elevated in BALF from patients with early IPF.

Conclusion Expression of SOX2 and MUC5B and lack of SPDEF in atypically differentiated cells of bronchiolised distal airspaces are consistent with abnormal programming of airway epithelial cells in IPF. NRG1 $\alpha$ may contribute to bronchiolisation of the distal lung seen in IPF.

\section{INTRODUCTION}

Idiopathic pulmonary fibrosis (IPF) is a lethal disease affecting 6.8-16.3 per 100000 individuals annually in the USA. ${ }^{1}$ While the mechanisms resulting in the loss of lung structure and function in this disorder remain poorly understood, there is increasing evidence that chronic injury to the respiratory epithelium activates aberrant repair processes, resulting in replacement of peripheral lung tissue with fibrotic scars and cysts lined by abnormal epithelial cells. These concepts are

\section{Key messages}

\section{What is the key question?}

- Abnormal differentiation of the respiratory epithelium is a feature of idiopathic pulmonary fibrosis (IPF). In this work, pathways governing mucus cell differentiation were investigated in lung tissues from patients with IPF and other chronic pulmonary disorders.

What is the bottom line?

- Mucus cells lining abnormal airways from patients with IPF expressed MUC5B and SOX2 but lacked SAM pointed domain containing ETS transcription factor (SPDEF) and forkhead box A3(FOXA3). Neuregulin $1 \alpha$, which drives mucus cell differentiation in vitro, was expressed in normal airway submucosal glands and in lungs from patients with IPF.

\section{Why read on?}

- Activation of abnormal respiratory epithelial differentiation programs may contribute to the expression of MUC5B and bronchiolisation of the distal lung, a salient feature of IPF.

- In contrast to findings in chronic obstructive pulmonary disease and $\mathrm{T}$ helper 2-driven mucus metaplasia in which SPDEF, FOXA3, and MUC5AC are expressed at high levels, MUC5B was the predominant mucin and neither SPDEF nor FOXA3 were detected in the abnormal mucus cells characteristic of the IPF lesions.

supported by experimental models in which epithelial cell injury ${ }^{2}$ or direct activation of remodelling processes leads to pulmonary fibrosis. ${ }^{3}$ Knowledge about the mechanisms influencing the differentiation and function of the respiratory epithelium in IPF may provide opportunities to diagnose, prevent or treat this incurable disorder.

Histopathological studies of IPF lungs reveal the typical 'usual interstitial pneumonia' pattern, with epithelial hyperplasia and apoptosis overlaying areas of scarring with fibroblast foci. In a focal manner, the alveolar architecture is destroyed, but regular lung structure may be found in adjacent regions. Bronchiolar abnormalities, including bronchiolisation of enlarged alveolar ducts, cysts and alveoli, are commonly seen, resulting in honeycombing when dilated airspaces communicate with proximal airways. Such bronchiolar changes are 
a salient feature of the disease. ${ }^{4} 5$ Recent data support the concept that bronchiolar abnormalities in IPF may be caused by cell autonomous changes in epithelial cell differentiation. Nuclear localisation of $B$-catenin, indicating activation of the Wnt pathway, has been reported in bronchiolar-like epithelia lining the conducting airways in patients with IPF. ${ }^{6}$

An intriguing feature of the bronchiolar-like epithelium lining honeycombing regions in the IPF lung is the presence of mucinladen cells, as mucus cells are normally absent from the distal lung. ${ }^{7}$ Moreover, while inflammation seems to be the main force inducing mucus cell differentiation in diseases such as asthma, cystic fibrosis, and chronic obstructive pulmonary disease (COPD), inflammation is usually mild in IPF. $^{5}$ These discrepancies led us to hypothesise that the mechanisms governing mucus cell metaplasia in IPF may be distinct, and that their identification may provide insight into processes regulating cell fate and airway epithelial function in IPF. Mucus cells in the respiratory tract are thought to originate from the reversible differentiation of basal, secretory or ciliated epithelial cells. During development, activation of the $\mathrm{Wnt} / \mathrm{B}$-catenin ${ }^{8}$ and Notch $^{9}$ pathways increases mucus cell numbers in the airway. Postnatally, mucus cell differentiation of SOX2-expressing airway epithelial cells ${ }^{10}$ is strongly driven by the epidermal growth factor receptor (EGFR or ErbB1) and T helper 2 (Th2) cytokines, which activate the transcription factors forkhead box A3 (FOXA3) and SAM pointed domain containing ETS transcription factor (SPDEF) in association with downregulation of FOXA2. ${ }^{11} 12$ Recently, activation of other members of the ErbB family by neuregulin-1 (NRG1) splice variants NRG1 $\alpha$ and NRG1 $\beta$ was shown to enhance transcription of mucin genes and to increase the number of mucus cells in vitro. ${ }^{13} 14$ In the gut, SPDEF, $^{15}$ Notch/AtoH ${ }^{16}$ and Krüppel-like factor 4 (KLF-4) ${ }^{17}$ transcriptionally influence mucus cell differentiation. Airway mucus cell differentiation is also influenced by site-specific cues. While MUC5B is the predominant mucin produced in submucosal glands, mucus cells lining conducting airways generally express MUC2 and MUC5AC ${ }^{18}$ and do not express MUC5B at high levels.

In the present study, the mechanisms governing mucus cell differentiation were assessed in the lungs of patients with IPF, in patients free of lung disease and in patients with other chronic lung disorders. The expression of transcription factors, ligands and/or activation markers indicating activity of the SPDEF, Notch, Erb2/3/4, and KLF-4 pathways was determined.

\section{MATERIALS AND METHODS Lung samples}

Anonymous lung samples were obtained at the University of Vienna and the University of Giessen Lung Center, at the time of lung transplantation from patients with IPF $(n=20)$, COPD $(\mathrm{n}=13)$, and with idiopathic pulmonary arterial hypertension (IPAH, $\mathrm{n}=5$ ); donor lobes resected because of size incompatibility served as controls $(n=6)$. Bronchial samples were obtained during lung cancer resection surgery at the University Hospital in Cincinnati. Patient characteristics are reported in online table S1. IPF was diagnosed according to American Thoracic Society/ European Respiratory Society (ATS/ERS) criteria. ${ }^{19}$ Utilisation of human lung samples was reviewed and approved by the Ethics Committee of Justus-Liebig-University of Giessen.

\section{Immunohistochemistry}

Five micrometre sections were deparaffinised and endogenous peroxidases were blocked. After antigen retrieval in $0.1 \mathrm{M}$ citrate
$(\mathrm{pH} 6)$ at $95^{\circ} \mathrm{C}$, sections were blocked with non-specific sera and primary antibodies (online table S2) were incubated overnight at $4^{\circ} \mathrm{C}$. Non-immune immunoglobins were used as negative controls. The HES1 ${ }^{20}$ and SPDEF ${ }^{21}$ antibodies were gifts from Dr Stanger (University of Pennsylvania) and Dr Watson (University of South Carolina). Biotinylated secondary antibodies and biotin-conjugated horseradish peroxidase complexes (both from Vector Laboratories, Burlingame, California, USA) were sequentially applied and staining was developed with nickeldiaminobenzidine (Sigma, St Louis, Missouri, USA). Staining by the NRG1 $\alpha$ (RB277) antibody was completely blocked when this antibody was pre-incubated with recombinant human NRG1 $\alpha$ EGF-like domain (296-HR, R\&D Systems, Minneapolis, Minnesota, USA). Sections were counter-stained with Alcian Blue and Nuclear Fast Red (Poly Scientific, Bay Shore, New York, USA) and examined at $\times 100$ magnification. Mucus cells were identified by Alcian Blue staining of the cytoplasm. The number of cells expressing MUC2, MUC5B, MUC5AC, FOXA3, SPDEF and HES1 was counted on each whole section and was expressed as a percentage of the total number of mucus cells on the section.

\section{Dual labelling to identify NRG1 $\alpha$ and p63}

After NRG1 $\alpha$ staining, sections were incubated with a mouse monoclonal antibody recognising p63 (sc-56188, Santa Cruz Biotechnology, Santa Cruz, California, USA), exposed to an alkaline phosphatase-conjugated goat anti-mouse antibody (31320, Thermo Scientific, Waltham, Massachusetts, USA), developed with Fast Red TR/Naphthol AS-MX (Sigma), and counterstained with haematoxylin.

\section{Bronchoalveolar lavage fluid samples}

Bronchoalveolar lavage fluid (BALF) was obtained at Bichat University Hospital (Paris, France) at the time of initial diagnostic evaluation in patients with IPF $(n=20)$, in patients free of interstitial lung disease $(\mathrm{n}=9)$, and in patients with non-IPF chronic interstitial pneumonias $(n=13)$. Patients gave informed consent and the protocol was approved by the ethics committee of HôtelDieu Hospital (Paris, France). Patient characteristics are reported in online table S3. BALF was concentrated fivefold with Amicon Ultra 3K columns (Millipore, Billerica, Massachusetts, USA).

\section{NRG1 $\alpha$ and NRG1 $\beta$ ELISA}

Plates were coated with a goat anti-NRG1 $\alpha$ capture antibody (AF296, R\&D Systems) and blocked with albumin. Standards were prepared with human NRG1 $\alpha$ EGF-like domain. A rabbit anti-NRG1 $\alpha$ detection antibody (RB277, Thermo Scientific) was applied followed by a peroxidase-conjugated anti-rabbit antibody (\#401315, Calbiochem, Gibbstown, New Jersey, USA). Tetramethylbenzidine (Sigma) was used as a substrate. NRG1 $\beta$ levels were determined with a commercial ELISA (DY377, R\&D Systems).

\section{Statistical analysis}

Comparisons between multiple groups were performed using Kruskall-Wallis' analysis of variance test followed by two-by-two comparisons with Mann-Whitney's U-test when a difference was detected. Immunohistochemical and BALF data were illustrated as box plots showing the 10th, 25th, 50th, 75th and 90 th percentiles. Clinical data were presented as meanIstandard error of the mean (SEM). Correlations between continuous variables were determined using Spearman's correlation test and linear regression. Associations between categorical variables were assessed using the $\chi^{2}$ test. A p value less than 0.05 was considered significant. 
Figure 1 Alcian blue, SOX2, and TTF1 staining in the distal airway epithelium. Tissue sections from organ donors $(n=7)$ and patients with chronic obstructive pulmonary disease (COPD) $(n=13)$, idiopathic pulmonary arterial hypertension (IPAH) $(n=5)$ and idiopathic pulmonary fibrosis (IPF) $(\mathrm{n}=20)$ were stained for SOX2 and TTF1 (brown/black colour), and counter-stained with Nuclear Fast Red

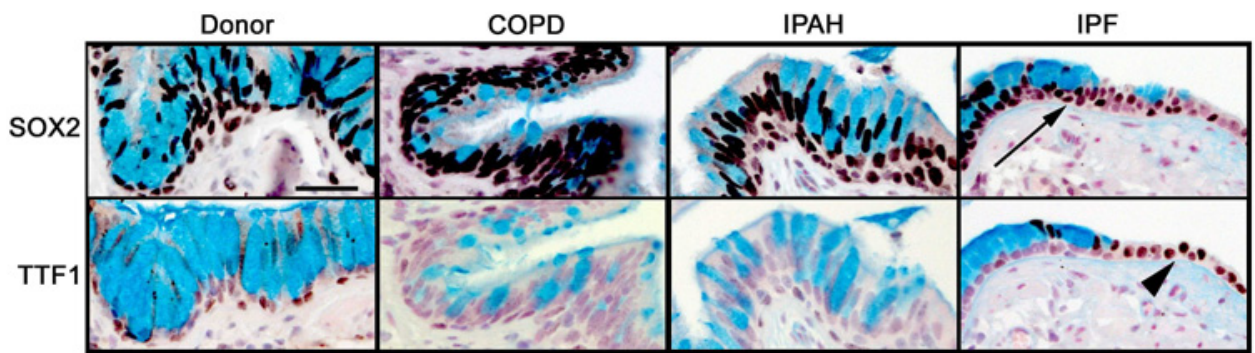
and Alcian Blue, the latter staining mucus cells. Airway mucus cells expressed SOX2 (arrow) and lacked TTF1 which was expressed by neighbouring epithelial cells in the lung with IPF (arrowhead). Serial sections are shown. Scale bar: $30 \mu \mathrm{m}$.

\section{RESULTS}

\section{Distinct morphology of the peripheral bronchiolar epithelium} in IPF

In the lungs of organ donors and patients with COPD and IPAH, airways were lined by a pseudostratified epithelium in which mucus cells were interspersed with basal, ciliated, and serous cell types. Differentiated mucus cell morphology was readily observed. In sharp contrast, peripheral bronchioles in honeycombed areas in lungs from patients with IPF were lined by mucus cells that were generally of low columnar morphology and usually organised as a homogenous single-layered epithelium. Such morphological appearance was observed in $53.5 \%$ of patients, and was never observed in donor, COPD or IPAH lungs $\left(\mathrm{p}<0.0001, \chi^{2}\right.$ test $)$

\section{Mucus cells lining bronchiolar lesions in IPF expressed SOX2} SOX2 is a transcription factor expressed selectively in conducting airway epithelial cells but not in alveolar regions. ${ }^{22}$ SOX2 drives proliferation and differentiation of the respiratory epithelium towards conducting airway epithelial cell types. ${ }^{23} 24$ SOX2 was detected in epithelial cells lining conducting airways in lungs from controls and patients with COPD, IPAH, and IPF (figure 1 and online figure s1). Mucus cells lining the honeycombed regions of lungs from patients with IPF were uniformly stained by the antibody against SOX2, consistent with cells lining conducting airways, but not alveolar regions. TTF1, a transcription factor expressed strongly in alveolar type II cells and at lower levels in conducting airways, was neither detected in mucus cells from IPF nor from patients without IPF. In contrast to the lack of TTF1 in honeycombed regions, TTF1 was highly expressed in hyperplastic alveolar epithelial cells in patients with IPF.

\section{Distinct mucin expression profile in IPF}

Mucus cells in lungs from donors and patients with COPD and IPAH expressed MUC2, MUC5AC, and MUC5B (figure 2 and online figure s2). In contrast, mucus cells lining IPF lesions expressed MUC5B, and rarely MUC2 or MUC5AC ( $p=0.018$ and $\mathrm{p}=0.0014$ in comparison to controls, respectively).

\section{Activation of the SPDEF differentiation pathway was lacking in IPF mucus cells}

Mucus cell differentiation in adult airways is influenced by Th2 cytokine-driven mechanisms controlled by the transcription factor SPDEF. Recent studies demonstrated that SPDEF is both necessary and sufficient to induce mucus cell differentiation in mice, and is strongly expressed in the lungs of patients with cystic fibrosis or after chronic smoking. ${ }^{11}$ SPDEF activates MUC5AC and FOXA3 following Th2 cytokine activation. While the mucus cells in patients with COPD, IPAH, and controls
Figure 2 Distinct mucin expression profile in idiopathic pulmonary fibrosis (IPF). Sections from organ donors $(n=7)$ and patients with chronic obstructive pulmonary disease (COPD) $(n=13)$, idiopathic pulmonary arterial hypertension (IPAH) $(n=5)$ and IPF $(n=20)$ were immunostained for mucins (black colour) and counter stained with Nuclear Fast Red and Alcian Blue. MUC2, MUC5AC, and MUC5B immunostaining are shown in figure $2 \mathrm{~A}$. Scale bar: $30 \mu \mathrm{m}$. Serial sections are shown. The percentage of mucus cells expressing MUC2, MUC5AC and MUC5B in the lungs are shown in figure 2B. Box plots show 10th, 25th, 50th, 75th and 90th percentiles. ${ }^{*} \mathrm{p}<0.05$ compared with donors.

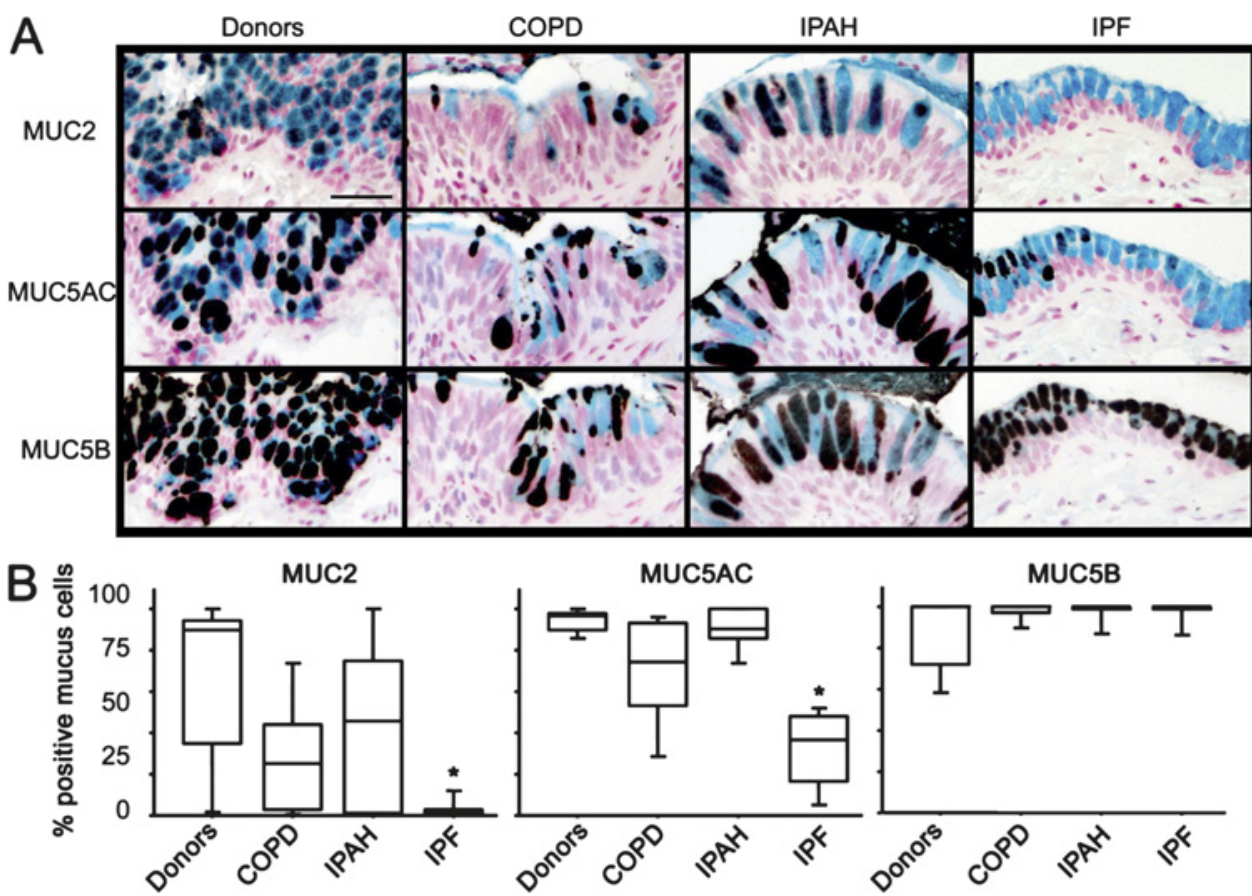


Figure 3 Lack of SPDEF and FOXA3 staining in idiopathic pulmonary fibrosis (IPF). Sections from organ donors $(n=7)$ and patients with chronic obstructive pulmonary disease (COPD) $(n=13)$, idiopathic pulmonary arterial hypertension (IPAH) $(n=5)$ and IPF $(n=20)$ were immunostained for FOXA3, SPDEF (serial sections), and HES1 (brown/black colour) and counter stained with Nuclear Fast red and Alcian blue as shown in figure $3 \mathrm{~A}$. Scale bar: $30 \mu \mathrm{m}$. Arrowheads show FOXA3positive nuclei. The percentage of mucus cells expressing FOXA3, SPDEF and HES1 in the lungs is shown in figure $3 \mathrm{~B}$. Box plots show 10th, 25th, 50th, 75th and 90th percentiles. ${ }^{*} p<0.05$ compared with donors.

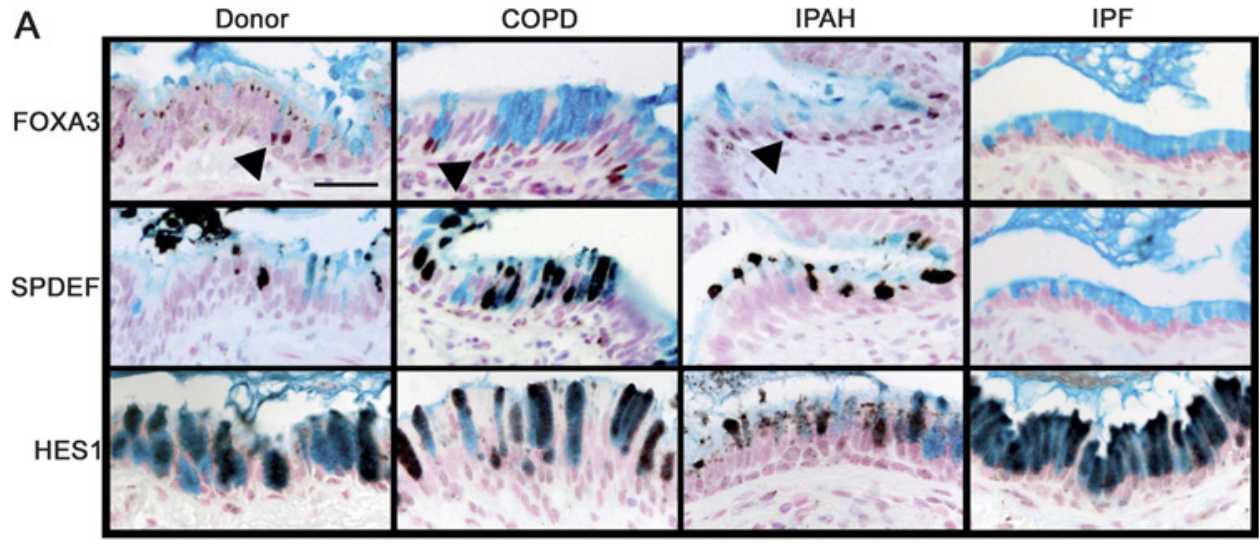

B

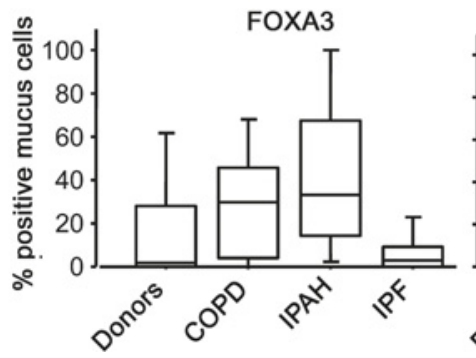

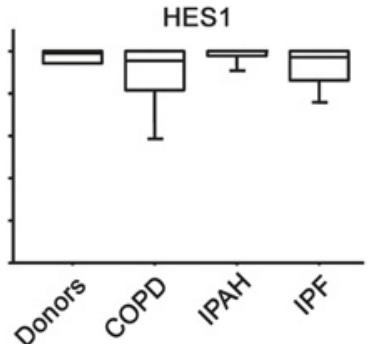

frequently stained with antibodies against FOXA3 and particularly SPDEF (figure 3 and online figure s3), the mucus cells in patients with IPF rarely stained for SPDEF $(p=0.0014$ in comparison to controls) or FOXA3, indicating that mucus cell differentiation in IPF likely occurs by distinct processes. Supporting this hypothesis, the transcription factor FOXA2, which is down-regulated by SPDEF, was frequently detected in the nuclei of mucus cells of patients with IPF (not shown). Staining for SPDEF was correlated with the expression of MUC5AC in controls and patients with COPD and IPAH (Spearman's test: $\mathrm{p}=0.0047$, figure 4 ).

Mucus cells from all groups of patients were selectively stained by an antibody against HES1, indicating Notch pathway activation (figure 3). KLF-4, which is expressed at the mRNA level in the lungs of patients with rapidly progressive $\mathrm{IPF}^{25}$ was not detected in lung tissue from any patient groups, although

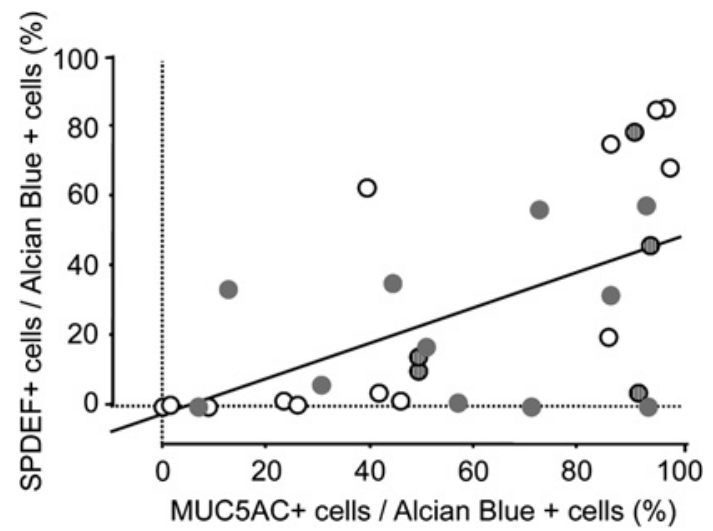

Figure 4 Correlation between MUC5AC and SPDEF. MUC5AC staining was correlated with that for SPDEF. Linear regression graph showing association between the percentage of mucus (Alcian blue positive) cells expressing SPDEF and MUC5AC in the lungs of organ donors (empty dots) and patients with chronic obstructive pulmonary disease (COPD) (grey dots), idiopathic pulmonary arterial hypertension (IPAH) (striped dots). $p=0.0015, R^{2}=0.291$. it was detected in mucus cells in human colonic epithelia (data not shown).

\section{NRG1 $\alpha$ was expressed in airway epithelial cells in IPF}

NRG1 $\alpha$ and NRG1ß induce mucus cell differentiation in human airway epithelial cells in vitro. ${ }^{14}$ The mucin expression profile of cells treated with NRG1 $\alpha$, but not NRG1ß, is similar to those in the IPF samples currently studied. While NRG1a staining was rarely and sporadically detected in tissue from patients with COPD (15\%) or IPAH (16\%), NRG1 $\alpha$ was readily detected in a majority of patients with IPF $(56 \%, p=0.0002$, $\chi^{2}$ test) where it was focally present in the cytoplasm and plasma membranes in a subset of epithelial cells lining bronchiolised regions (figure 5 and online figure s4). NRG1ß was not detected in normal submucosal glands or in airways or alveolar regions of tissues from either donors or patients with COPD, IPAH or IPF, but was readily detected in human tonsils (not shown).

\section{Association of NRG1 $\alpha$ with p63}

NRG1 $\alpha$-stained epithelial cells in the lungs of patients with IPF were generally squamous in morphology. Double immunofluoresence demonstrated that the NRG1 $\alpha$-positive cells were generally located in close proximity to p63-positive cells within pseudostratified/stratified regions of the bronchiolised epithelium in IPF (figure 6 and online figure s5).

\section{MUC5B and NRG1 $\alpha$ were expressed in normal proximal airway submucosal glands}

In striking similarity to the distal lung of patients with IPF mucus cells in normal proximal airway submucosal glands expressed MUC5B but undetectable levels of MUC5AC, while NRG1 $\alpha$ was detected in serous cells, in close proximity to p63-positive cells (figure 7).

\section{Increased levels of NRG1 $\alpha$ in BALF in early-stage IPF}

To determine whether increased epithelial NRG1 $\alpha$ expression was an early event in IPF, we compared BALF NRG1 $\alpha$ and NRG1 $\beta$ levels in patients with early IPF, in patients with other 
Figure 5 NRG1 $\alpha$ staining in idiopathic pulmonary fibrosis (IPF) lung tissue. Lung sections from organ donors $(n=7)$ and patients with chronic obstructive pulmonary disease (COPD) $(n=13)$, idiopathic pulmonary arterial hypertension (IPAH) $(n=5)$ and IPF $(n=20)$ were immunostained for NRG1 $\alpha$ and NRG1 $\beta$ (black colour) and counter stained with Nuclear Fast Red and Alcian Blue. The mucus cell differentiation factor NRG1 $\alpha$ was detected in a subset of airway epithelial cells in patients with IPF (arrow); these cells did not express NRG1 $\beta$ (arrowhead). Serial sections are shown. Scale bar: $30 \mu \mathrm{m}$.

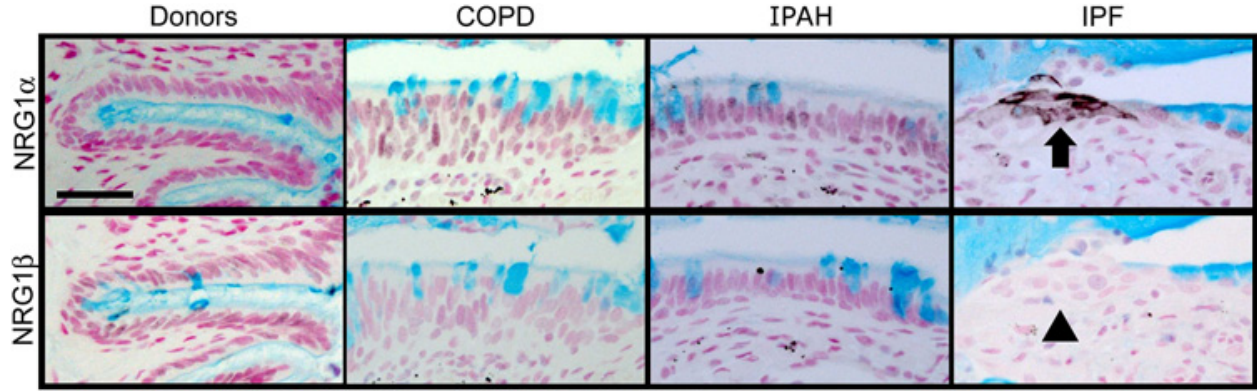

chronic interstitial lung disease and in controls. BALF NRG1 $\alpha$ levels were higher in patients with IPF $(29.2 \pm 4.6 \mathrm{nM})$ compared with controls $(14.2 \pm 2.9 \mathrm{nM}, \mathrm{p}=0.021)$ and with patients with non-IPF chronic interstitial lung disease $(21.6 \pm 7.9 \mathrm{nM}$, $\mathrm{p}=0.043)$. NRG1 $\beta$ levels were similar in all groups of patients (figure 8).

\section{DISCUSSION}

There is increasing evidence that chronic alveolar type II cell injury, whether related to endoplasmic reticulum ${ }^{26}$ or lysosomal ${ }^{27}$ stress, is involved in the pathogenesis of IPF. Recurrent epithelial cell death induces a regenerative response associated with activation of embryologically important pathways such as Wnt/ $\beta$-catenin. ${ }^{6}$ Fibroblast foci develop progressively and a marked distortion of alveolar structure occurs. Septal structures are replaced by enlarged distal airspaces or cysts typically

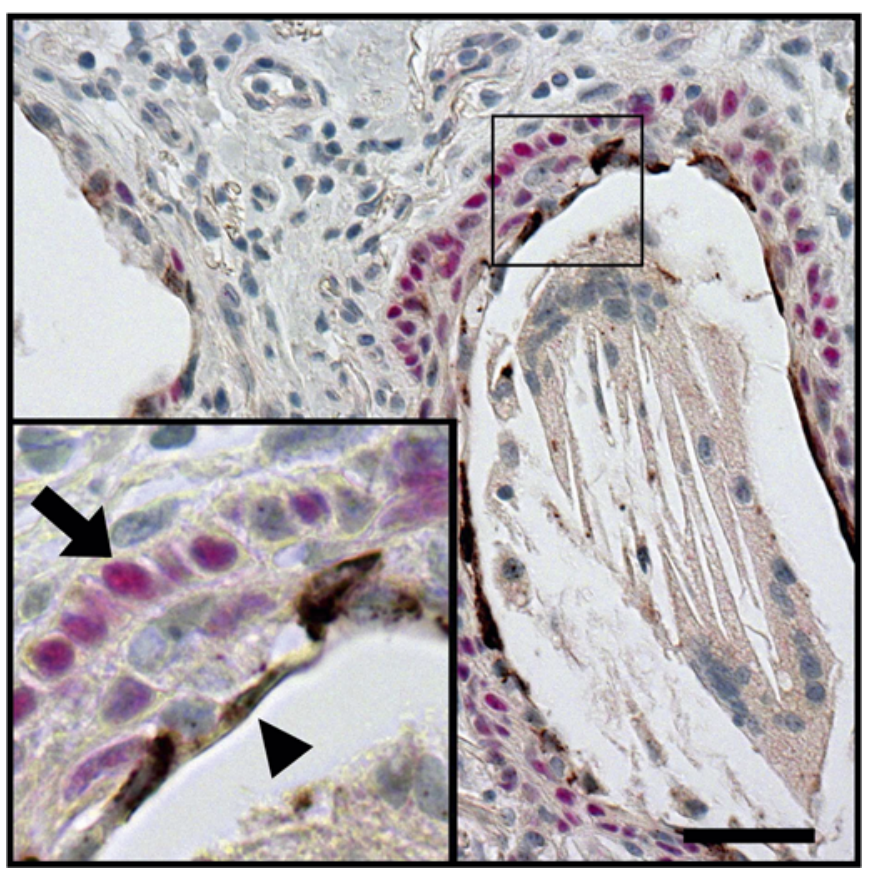

Figure 6 Cellular localisation of NRG1 $\alpha$ and p63 in the idiopathic pulmonary fibrosis (IPF) lesions. Sections from patients with IPF were co-immunostained for NRG1 $\alpha$ (brown color) and p63 (red color) and counterstained with haematoxylin. Squamous NRG1 $\alpha$-positive cells (arrowheads) were localised in close apposition to p63-positive basal cells (arrows) in the distal lung of patients with IPF. Image is representative of $n=12$ experiments. Scale bar: $50 \mu \mathrm{m}$. covered by epithelial cells with characteristics of proximal airway epithelium. Expression of SOX2 and paucity of TTF1 in epithelial cells in the bronchiolised distal airspaces in IPF support this concept.

In IPF, the airways were often lined by atypical, cuboidal mucus cells. In contrast to the abundant expression of SPDEF, MUC5AC and MUC2 by mucus cells in COPD and other chronic lung diseases, mucus cells in IPF expressed MUC5B, a mucin expressed at higher levels in submucosal glands, and lacked SPDEF, implicating the activation of specific cell differentiation pathways. NRG1 $\alpha$ expression was detected in the bronchiolised distal airspaces in IPF as well as in normal airway submucosal glands, supporting the concept that NRG1 $\alpha$ influences a mucus cell differentiation program distinct from that seen in the surface airway epithelium of patients with COPD or cystic fibrosis. ${ }^{11}$

In the airways of organ donors as well as patients with COPD and IPAH, expression of SPDEF by mucus cells was correlated with expression of MUC5AC, further supporting the concept of a causal association between SPDEF expression and mucus metaplasia. Interestingly, as seen in mouse models, ${ }^{11} 12$ SPDEF was prominent in the cytoplasm of mucus cells. It is unclear whether SPDEF functions solely as a nuclear transcription factor or influences mucus cell differentiation via mechanisms that do not require direct activation of target genes. ${ }^{12}$ FOXA3 was infrequently detected in mucus cells of patients with and without IPF. In line with the finding that FOXA3 expression is not associated with airway mucus metaplasia in patients with asthma, ${ }^{28}$ this result suggests that FOXA3 may not play a critical role in mucus metaplasia in humans.

NRG1 $\alpha$, which enhances MUC5B but not MUC5AC expression in human airway epithelial cells, was expressed in the lungs of $56 \%$ of patients with IPF, being localised in squamous epithelial cells located in close proximity to basal cells expressing p63 (figure 6). NRG1 $\alpha$ may also be expressed by other squamous epithelia. The lack of NRG1 $\alpha$ detection in the remaining patients may have been related either to absent NRG1 $\alpha$ expression or, as NRG1 $\alpha$ was focally expressed in IPF lungs, to sampling of areas of the lung not expressing NRG1 $\alpha$. NRG1 $\alpha$ belongs to a family of signalling proteins that exert their effects through the EGFR-related receptors ErbB2, ErbB3 and ErbB4 NRG1 is a large and complex gene from which multiple protein isoforms are produced using several promoters and splicing; $\alpha$ and $\beta$ isoforms of NRG1 differ in their EGF-like domain. While the NRG1 gene is necessary for heart and brain development, ${ }^{29}$ mice carrying a deletion of NRG1 $\alpha$ EGF-like domain develop normally. $^{30}$ 

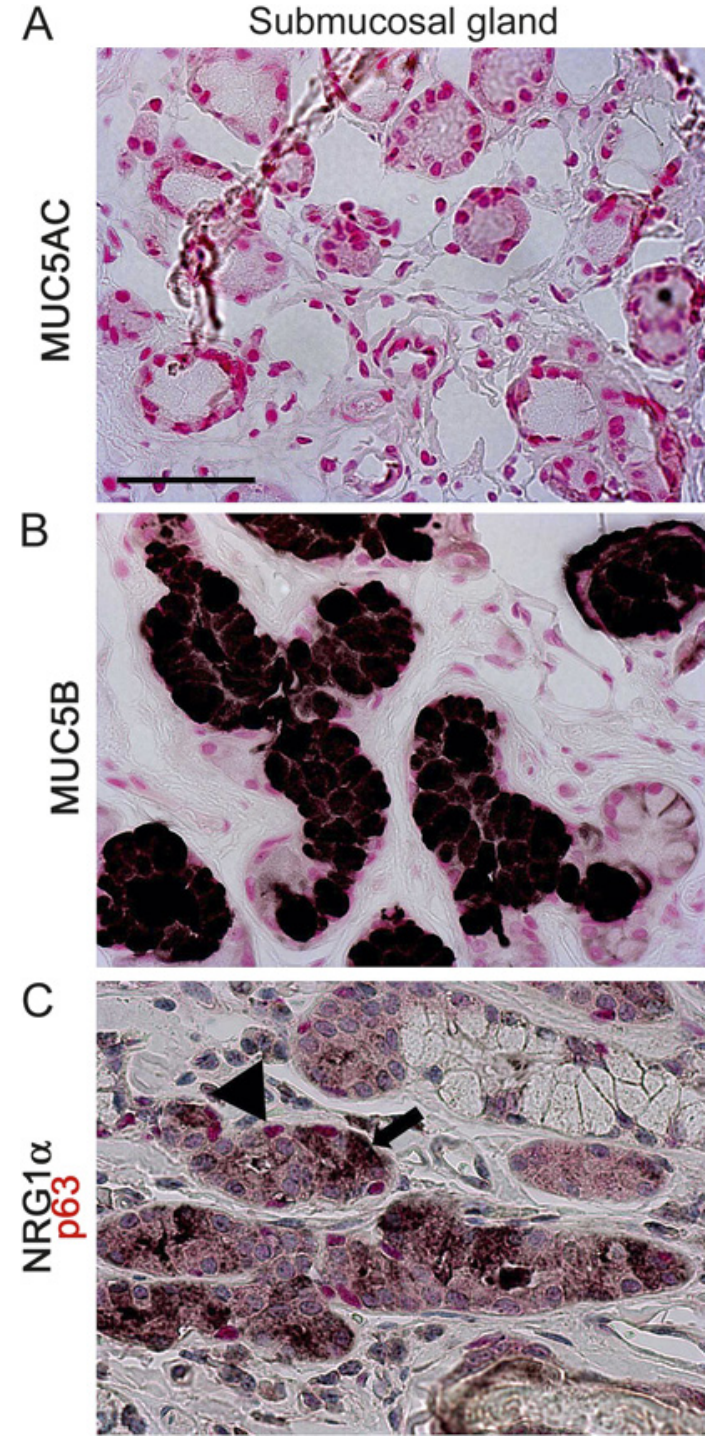

Figure 7 Normal proximal airway submucosal glands share common features with the peripheral lung lesion in idiopathic pulmonary fibrosis (IPF) patients. Normal submucosal gland sections were immunostained for MUC5AC (black, figure 7A), MUC5B (black, figure 7B), and co-stained for NRG1 $\alpha$ (brown) and p63 (red, figure 7C), with Nuclear Fast Red counterstaining. Normal proximal airway submucosal gland mucus cells expressed MUC5B but not MUC5AC. NRG1 $\alpha$-expressing serous cells (arrows) were apposed to p63-positive basal cells (arrowheads). Photomicrographs representative of two patients. Scale bar: $50 \mu \mathrm{m}$.

The fact that high levels of NRG1 $\alpha$ were detected in the BALF of patients with IPF at the time of diagnosis suggests that increased expression of this factor is an early event in IPF. However, age of patients may be a confounding factor in the present study because patients with IPF were older. We could not study BALF NRG1 $\alpha$ expression in the same patients who were included in the immunohistochemical study. In transgenic mice, activation of ErbB1 causes pulmonary fibrosis, ${ }^{3}$ while silencing of ErbB3 signalling in the lung protects mice against bleomycin-induced lung fibrosis. ${ }^{31}$ While the present studies support a potential role of NRG1 $\alpha$ signalling in IPF, the mechanisms underlying differentiation, bronchiolarisation, and fibrosis are likely to be complex; signalling by the extracellular matrix may be important and other signalling molecules may be
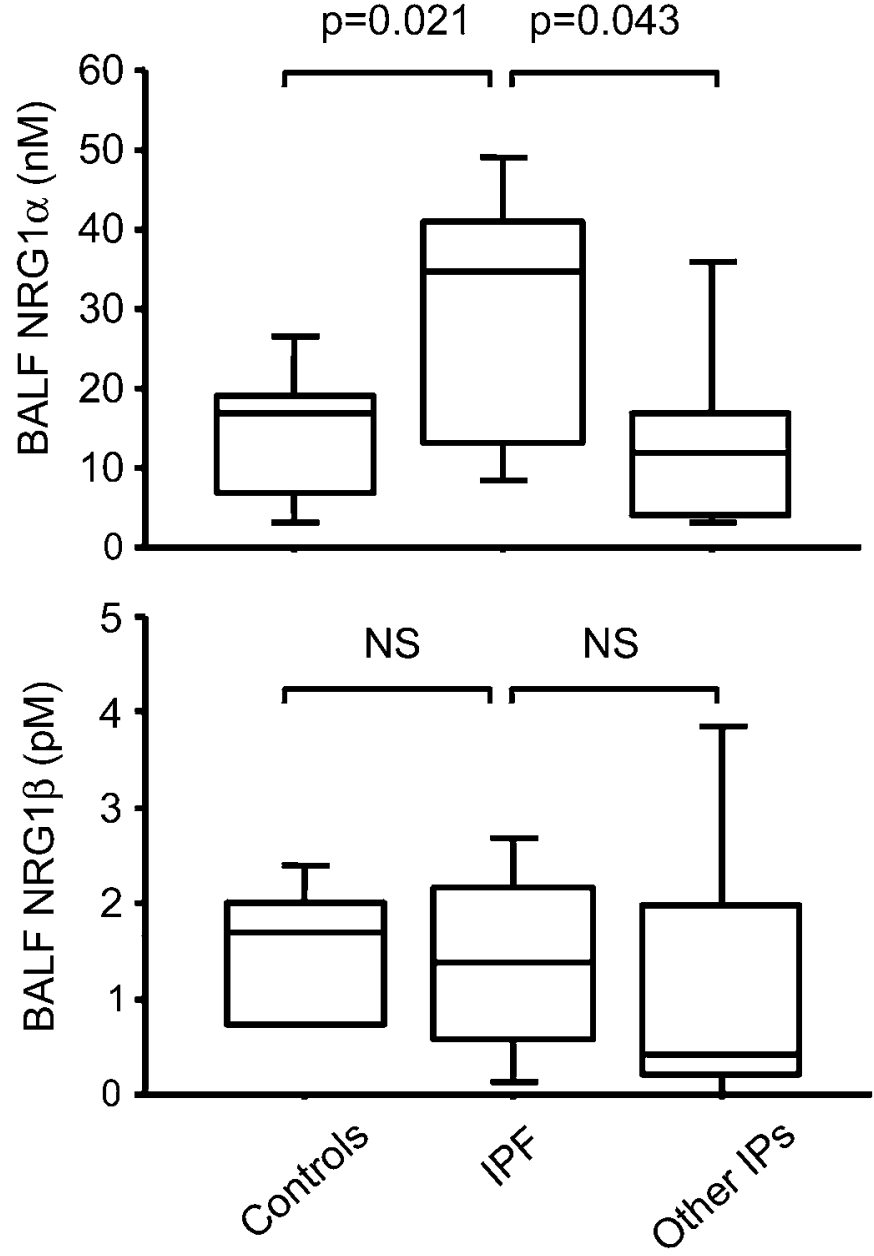

Figure 8 Quantitation of NRG1 $\alpha$ in bronchoalveolar lavage fluid (BALF) in early stage idiopathic pulmonary fibrosis (IPF). Increased levels of NRG1 $\alpha$ in BALF of patients with early-stage IPF. NRG1 $\alpha$ and NRG1ß concentrations determined by ELISA in BALF of patients free of chronic lung disease, in patients with early IPF and in patients with other chronic interstitial pneumonias (IPs). Box plots show 10th, 25th, 50th, 75th and 90th percentiles.

implicated. In patients with diffuse panbronchiolitis, polymorphisms of the MUC5B promoter are associated with increased promoter activity, increased MUC5B expression, and disease severity, suggesting that the differentiation of mucus cells towards a MUC5B+/MUC5AC- phenotype in IPF may be influenced genetically. ${ }^{32}$

Whether the acquisition of a mucus cell phenotype in the lungs of patients with IPF is an active differentiation process was not addressed in this study. Deletion of Munc13-2, a gene encoding an exocytic priming protein, causes the accumulation of MUC5B in the cytoplasm of Clara cells in mice; ${ }^{33}$ alterations of exocytic pathways may contribute to the acquisition of a mucus cell phenotype in the bronchiolised regions of IPF lungs. Cytoplasmic expression of HES1 was a constant feature of mucus cells, suggesting a role for the Notch pathway in mucus cell differentiation in the adult respiratory tract, although HES1 expression is also regulated by Notch-independent mechanisms. $^{34}$

KLF-4, which regulates mucus cell differentiation in the gut, is highly expressed in the lungs of patients with rapidly progressive IPF. ${ }^{25}$ However, KLF-4 was not detected in the present study, suggesting that the mechanisms driving epithelial 
metaplasia in IPF may be specific to the respiratory system. Conversely, lack of KLF-4 detection in lungs with IPF may have been related to insufficient sensitivity of the antibody, post-translational regulation or selection bias.

Many features of the lesions seen in the peripheral lung in IPF were similar to those characteristic of proximal airway submucosal glands that express MUC5B rather than MUC5AC. ${ }^{18}$ NRG1 $\alpha$ was found to be expressed in the cytoplasm of serous cells in submucosal glands, in proximity to p63-positive basal cells. P63-positive basal cells function as airway epithelial progenitor cells in humans. ${ }^{35}$ We speculate that NRG1 $\alpha$ expression seen in lungs of patients with IPF may influence abnormal epithelial differentiation in cells derived from basal or other progenitor cells, resulting in mucus metaplasia with cellular features more typical of submucosal glands mucus cells.

In conclusion, our findings indicate that mucus cell differentiation in the distal airway epithelium of patients with IPF is distinct from that typical of the SPDEF-associated pathways seen in COPD, asthma or cystic fibrosis. The finding that NRG1 $\alpha$ is increased in BALF in patients with IPF, and its role in regulation of mucus cell differentiation, ${ }^{14}$ support its potential paracrine effect influencing bronchiolisation and MUC5B expression.

Acknowledgements The authors thank Dr Kathryn Wikenheiser-Grokamp for helpful suggestions, David Loudy, Gail Macke and Paula Blair for excellent technical assistance, and Ann Maher for secretarial help.

Funding This work was supported by a travel grant from the Société de Pneumologie de Langue Française (LP), the National Institutes of Health HL095580 and HL090156, the Cystic Fibrosis Foundation RDP Center and the European Commission (FP7 grant agreement \#202224, European IPF Network). Other Funders: NIH.

Competing interests None.

Ethics approval This study was conducted with the approval of the Justus-Liebig University of Giessen.

Provenance and peer review Not commissioned; externally peer reviewed.

\section{REFERENCES}

1. Raghu G, Weycker D, Edelsberg J, et al. Incidence and prevalence of idiopathic pulmonary fibrosis. Am J Respir Crit Care Med 2006;174:810-16.

2. Moore BB, Hogaboam CM. Murine models of pulmonary fibrosis. Am J Physiol Lung Cell Mol Physiol 2008:294:L152-60.

3. Korfhagen TR, Swantz RJ, Wert SE, et al. Respiratory epithelial cell expression of human transforming growth factor-alpha induces lung fibrosis in transgenic mice. J Clin Invest 1994;93:1691-9.

4. Silva CI, Muller NL, Lynch DA, et al. Chronic hypersensitivity pneumonitis: differentiation from idiopathic pulmonary fibrosis and nonspecific interstitial pneumonia by using thin-section CT. Radiology 2008;246:288-97.

5. Song JW, Do KH, Kim MY, et al. Pathologic and radiologic differences between idiopathic and collagen vascular disease-related usual interstitial pneumonia. Chest 2009;136:23-30.

6. Chilosi M, Poletti V, Zamo A, et al. Aberrant Wnt/beta-catenin pathway activation in idiopathic pulmonary fibrosis. Am J Pathol 2003;162:1495-502.

7. Boers JE, Ambergen AW, Thunnissen FB. Number and proliferation of clara cells in normal human airway epithelium. Am J Respir Crit Care Med 1999:159:1585-91.

8. Mucenski ML, Nation JM, Thitoff AR, et al. Beta-catenin regulates differentiation of respiratory epithelial cells in vivo. Am J Physiol Lung Cell Mol Physiol 2005;289:L971-9.

9. Guseh JS, Bores SA, Stanger BZ, et al. Notch signaling promotes airway mucous metaplasia and inhibits alveolar development. Development 2009;136:1751-9.
10. Tompkins DH, Besnard V, Lange AW, et al. Sox2 is required for maintenance and differentiation of bronchiolar clara, ciliated, and goblet cells. PLoS One 2009:4:e8248.

11. Chen G, Korfhagen TR, Xu Y, et al. SPDEF is required for mouse pulmonary goblet cell differentiation and regulates a network of genes associated with mucus production. J Clin Invest 2009;119:2914-24.

12. Park KS, Korfhagen TR, Bruno MD, et al. SPDEF regulates goblet cell hyperplasia in the airway epithelium. J Clin Invest 2007;117:978-88.

13. Vermeer PD, Panko L, Karp P, et al. Differentiation of human airway epithelia is dependent on erbB2. Am J Physiol Lung Cell Mol Physiol 2006;291:L175-80.

14. Kettle R, Simmons J, Schindler F, et al. Regulation of neuregulin 1beta1-induced MUC5AC and MUC5B expression in human airway epithelium. Am J Respir Cell Mol Biol 2010;42:472-81

15. Noah TK, Kazanjian A, Whitsett J, et al. SAM pointed domain ETS factor (SPDEF) regulates terminal differentiation and maturation of intestinal goblet cells. Exp Cell Res 2010;316:452-65.

16. Shroyer NF, Helmrath MA, Wang VY, et al. Intestine-specific ablation of mouse atonal homolog 1 (Math1) reveals a role in cellular homeostasis. Gastroenterology 2007; 132:2478-88

17. Katz JP, Perreault N, Goldstein BG, et al. The zinc-finger transcription factor Klf4 is required for terminal differentiation of goblet cells in the colon. Development 2002:129:2619-28.

18. Thai $\mathbf{P}$, Loukoianov $A$, Wachi $S$, et al. Regulation of airway mucin gene expression. Annu Rev Physiol 2008; 70:405-29.

19. Anon; American Thoracic Society. Idiopathic pulmonary fibrosis: diagnosis and treatment. International consensus statement. American Thoracic Society (ATS) and the European Respiratory Society (ERS). Am J Respir Crit Care Med 2000:161:646-64.

20. Ito T, Udaka N, Yazawa T, et al. Basic helix-loop-helix transcription factors regulate the neuroendocrine differentiation of fetal mouse pulmonary epithelium. Development 2000;127:3913-21.

21. Feldman RJ, Sementchenko VI, Gayed M, et al. Pdef expression in human breast cancer is correlated with invasive potential and altered gene expression. Cancer Res 2003:63:4626-31.

22. Gontan C, de Munck A, Vermeij M, et al. SOX2 is important for two crucial processes in lung development: branching morphogenesis and epithelial cell differentiation. Dev Biol 2008:317:296-309.

23. Lu Y, Futtner C, Rock JR, et al. Evidence that SOX2 overexpression is oncogenic in the lung. PLOS One 2010:5:e11022.

24. Tompkins DH, Besnard V, Lange AW, et al. SOX2 activates cell proliferation and differentiation in the respiratory epithelium. Am J Respir Cell Mol Biol 2011:45:101-10

25. Boon K, Bailey NW, Yang J, et al. Molecular phenotypes distinguish patients with relatively stable from progressive idiopathic pulmonary fibrosis (IPF). PLoS One 2009; 4:e5134.

26. Korfei M, Ruppert C, Mahavadi P, et al. Epithelial endoplasmic reticulum stress and apoptosis in sporadic idiopathic pulmonary fibrosis. Am J Respir Crit Care Med 2008;178:838-46.

27. Mahavadi P, Korfei M, Henneke I, et al. Epithelial stress and apoptosis underlie Hermansky-Pudlak syndrome-associated interstitial pneumonia. Am J Respir Crit Care Med 2010;182:207-19

28. Park SW, Verhaeghe C, Nguyenvu LT, et al. Distinct roles of FOXA2 and FOXA3 in allergic airway disease and asthma. Am J Respir Crit Care Med 2009; 180:603-10.

29. Meyer D, Birchmeier C. Multiple essential functions of neuregulin in development Nature 1995:378:386-90

30. Li L, Cleary S, Mandarano MA, et al. The breast proto-oncogene, HRGalpha regulates epithelial proliferation and lobuloalveolar development in the mouse mammary gland. Oncogene 2002;21:4900-7.

31. Nethery DE, Moore BB, Minowada G, et al. Expression of mutant human epiderma receptor 3 attenuates lung fibrosis and improves survival in mice. J Appl Physiol 2005;99:298-307.

32. Kamio K, Matsushita I, Hijikata M, et al. Promoter analysis and aberrant expression of the MUC5B gene in diffuse panbronchiolitis. Am J Respir Crit Care Med 2005; 171:949-57.

33. Zhu Y, Ehre C, Abdullah LH, et al. Munc13-2-/- baseline secretion defect reveals source of oligomeric mucins in mouse airways. J Physiol 2008;586:1977-92.

34. Sang L, Roberts JM, Coller HA. Hijacking HES1: how tumors co-opt the antidifferentiation strategies of quiescent cells. Trends Mol Med 2010;16:17-26.

35. Rock JR, Onaitis MW, Rawlins EL, et al. Basal cells as stem cells of the mouse trachea and human airway epithelium. Proc Natl Acad Sci U S A 2009;106:12771-5. 